\title{
La reforma de la formación inicial del profesorado de ciencias de secundaria: propuesta de un diseño del currículo basado en competencias
}

\section{Reform of initial science teacher training: design proposal for a competency-based curriculum}

\author{
F. Javier Perales Palacios, J. Manuel Cabo Hernández, J. Miguel Vílchez González, Manuel Fernández González, Francisco \\ González García, Pilar Jiménez Tejada \\ Dpto. de Didáctica de las Ciencias Experimentales. Universidad de Granada \\ fperales@ugr.es,jmcabo@ugr.es, http://www.ugr.es/-jmvilchez/Web/, mfgfaber@ugr.es, http://www.ugr.es/local/pagoga, pjtejada@ugr.es
}

RESUMEN • El principal objetivo de este trabajo es proponer un mecanismo de selección de competencias específicas para la formación inicial del profesorado de ciencias experimentales, contando para ello con distintas fuentes relevantes para dicha formación. Con tal fin hemos hecho uso de un procedimiento consistente en ir incorporando de forma crítica las competencias procedentes de las siguientes fuentes curriculares: los modelos de formación inicial del profesorado vigentes en la literatura educativa, el currículo actual de ciencias en la educación secundaria obligatoria en España, el Programa internacional de evaluación PISA, la opinión del profesorado en activo mediante una encuesta cuyos resultados más relevantes son mostrados, las concepciones epistemológicas del profesorado, la profesionalización docente y las demandas que genera y, finalmente, la dimensión social de la ciencia.

PALABRAS CLAVE: didáctica de las ciencias experimentales; formación inicial del profesorado; educación secundaria; currículo; competencias.

SUMMARY • The primary objective of this paper is to propose a mechanism for selecting specific competences for preservice science teacher training, referring to relevant sources for such training. To this end we have used an trans-disciplinary procedure consistent with incorporating critical competences from the following curricular sources: Models of current preservice teacher training in educational literature, the current curriculum of Science in Compulsory Secondary Education in Spain, the Program for International Student Assessment (PISA), the views of in-service secondary school teachers (through a survey whose most relevant results are given), teachers' epistemological conceptions, the professionalization of teaching and the demands it generates and, finally the social dimension of science.

KEYWORDS: science education; preservice training teachers; high school; curriculum; competences.

Fecha de recepción: marzo 2012 • Aceptado: octubre 2012

Perales, F. J., Cabo, J. M., Vílchez, J. M., Fernández-González, M., González-García, F., Jiménez-Tejada, P. (2014). La reforma de la formación inicial del profesorado de ciencias: propuesta de un diseño del currículo basado en competencias. Enseñanza de las Ciencias, 32 (1), pp. 9-28 


\section{INTRODUCCIÓN}

La aparición periódica de los sucesivos Informes PISA ha ido generando en nuestro país un indudable impacto mediático ante la aparente degradación de nuestro sistema educativo, en general, y de determinadas parcelas de conocimiento, como es el caso de las ciencias, en particular. El progresivo respeto que esta prueba de evaluación ha ido alcanzando a nivel internacional ha permitido a los docentes disponer de un buen punto de partida como elemento de diagnóstico del proceso de enseńanza-aprendizaje. No obstante, resulta a todas luces ineludible el análisis de las causas que originan esta situación y la puesta en marcha de un plan de actuaciones que permita ir corrigiendo esta negativa tendencia.

Una de las causas que aparecen como factor común en los análisis que de los resultados de dicho informe se han publicado se refiere a la formación del profesorado. La situación española de esta trascendental parcela educativa, especialmente en el caso de la educación secundaria, ha resultado durante las últimas décadas cuando menos insuficiente y desfasada. Se trata de la única dimensión educativa institucional que ha permanecido inalterada desde la década de los setenta del pasado siglo hasta la época reciente.

Desde el curso académico 2009-2010 se ha implementado en España el denominado Máster Universitario en Profesorado (Ministerio de Educación y Ciencia, 2007a), el cual se enmarca dentro de la nueva estructura de las enseñanzas universitarias y del llamado Espacio Europeo de Educación Superior (EEES), con carácter de posgrado.

El objetivo de este trabajo es proponer un sistema de competencias específicas para la formación inicial del profesorado de ciencias, a partir de distintas fuentes relevantes para dicha formación, utilizando para ello un procedimiento metodológico transdisciplinar y que supone un punto de partida para el diseño de dicho currículo.

\section{EL CONCEPTO DE COMPETENCIA PROFESIONAL EN EL EEES}

Buscar el origen del concepto de competencia profesional resulta difícil, ya que el término posee una larga historia y diversos significados. Hay quien se remonta a los años sesenta del pasado siglo y a Chomsky como primer autor que utilizó el término, aunque es más frecuente referirlo a los años setenta con las investigaciones de McClelland en la Universidad de Harvard, en el marco de la formación de recursos humanos para las empresas.

La enorme variedad de definiciones ha provocado un esfuerzo en la delimitación conceptual (Tejada, 1999a; 1999b), debido a un doble problema: el carácter polisémico del término y la existencia de diversos enfoques, de modo que resulta complicado hablar de definiciones consensuadas universalmente. Lo que sí cabe hacer es identificar aquellas características que le son propias y que nos permiten diferenciarlo de otros conceptos. En este sentido, concretamos las siguientes especificaciones:

\section{Competencia es un concepto complejo que incluye e integra distintos tipos de saberes:}

Si competencia se define como un conjunto de conocimientos, procedimientos y actitudes combinados, coordinados e integrados para «saber hacer»y «saber estar» en el ejercicio profesional, el dominio de estos saberes permite ser «capaz de» actuar con eficacia en situaciones profesionales cambiantes. Pero ¿ser capaz es lo mismo que ser competente? Esta definición, quizá la más generalizada, no nos aclara la cuestión.

Las competencias implican el desarrollo de capacidades que constituyen conjuntos de saberes integrados en las competencias, pero ser capaz no implica necesariamente ser competente. Se puede ser 
capaz de desarrollar una técnica pero se puede también no ser competente a la hora de seleccionar qué técnica usar y cuándo en un contexto profesional concreto, como en nuestro caso, un aula.

\section{El núcleo central de los saberes en competencias es el saber hacer:}

Si se quiere dar prioridad o un papel especial al saber hacer, podría interpretarse como una confusión entre competencia y capacidad. Sin embargo, la competencia no reside solo en los recursos disponibles (capacidades), sino en la movilización misma de los recursos ante problemas y dificultades que surgen en el desempeño profesional.

\section{El desarrollo de competencias profesionales implica el contexto.}

Si las competencias no residen en los recursos (saber y saber hacer), sino en su utilización, solo son definibles en la acción, en donde la experiencia es un factor clave. Es decir, el saber y el saber hacer como productos de la formación no conseguirán desarrollar competencias si no se utilizan en el propio contexto profesional. En el caso del profesor de educación secundaria, un contexto de aula.

Este contexto de aula en el que el profesor de educación secundaria va a desarrollar su labor es enormemente cambiante, por el currículo, el ámbito socioeconómico del centro, el tipo de alumnado, el nivel educativo, etc., todo ello implica la selección de los recursos necesarios en cada situación específica. Sin desempeño profesional no hay desarrollo de competencias.

El comienzo de este siglo hereda esta situación de polisemia y falta de delimitación conceptual. Posteriormente, el modelo de competencias se ha popularizado en la universidad española, en gran medida debido al Proyecto Tuning (González y Wagenaar, 2003).

En el ámbito del EEES se pretende que la educación deba centrarse en la adquisición de competencias por parte del estudiante, es decir, será un proceso enfocado hacia el aprendizaje. Por tanto, se entienden las competencias profesionales como resultados de aprendizaje, para lo que se establecerán objetivos de enseñanza derivados del análisis de tales competencias. Sin embargo, el Proyecto Tuning puso de manifiesto la coincidencia por parte de académicos, empleadores y estudiantes en la valoración de las competencias genéricas y específicas que cualquier titulación de grado puede compartir, pero no realizaron análisis sobre titulaciones de posgrado ni se explicitaron los enfoques y modelos seguidos.

A la dificultad conceptual de las competencias profesionales se añade, pues, la existencia de diversos enfoques y modelos en los que se enmarcan, siendo frecuente que no se expliciten. Por lo general, el desarrollo de competencias obedece a la definición del perfil profesional y de las tareas y funciones del profesional.

\section{LAS COMPETENCIAS EN EL NUEVO MÁSTER DE PROFESORADO}

El CAP representó en su momento la primera regulación en España de una formación inicial del profesorado de educación secundaria, pero su permanencia durante casi cuatro décadas acabó convirtiéndolo en una iniciativa obsoleta y burocratizada. La formación proporcionada por este podría describirse como formación por yuxtaposición. Es decir, frente a un modelo de integración de la formación científico-didáctica en los estudios de grado (sea propiamente como grado de profesorado o como itinerario formativo en los grados de ciencias), se optó por cursar estudios de ciencias a nivel de grado y, posteriormente, hacerlo con la vertiente pedagógica de estos (CAP). Es decir, para ser profesor permanente de educación secundaria había que seguir el siguiente itinerario: 


\section{Licenciatura en Ciencias (Física, Química...) + CAP + Oposiciones}

Está claro que, dado el tiempo y las exigencias de cada uno de los dos primeros sumandos, en dicho itinerario subyacía la idea de que "para ser un buen profesor bastaba con dominar el contenido científico», ignorando la relevancia del "conocimiento didáctico del contenido» (Shulman, 1985; Kaya, 2009) y las propias exigencias de una sociedad en vertiginoso proceso de cambio que demanda cada día más «educadores» frente a especialistas en el contenido que se va a enseńar.

Ese presupuesto y los propios intereses de los gremios profesionales (universitarios y no universitarios) abortaron cualquier intento serio de modificar ese estado de cosas. Solo la nueva regulación de los grados y posgrados permitió encajar la formación inicial del profesorado de educación secundaria en la figura legal de máster (Ministerio de Educación y Ciencia, 2007a).

A pesar del avance que el máster ha supuesto frente al CAP, tal como su duración (curso completo frente a un trimestre), se ha seguido optando por una formación didáctica yuxtapuesta.

Asimismo, tanto el máster como el CAP optaron por la fragmentación del currículo en una secuencia temporal que podría representarse como:

\section{Formación psico-socio-pedagógica + Didáctica específica + Prácticas de enseñanza}

A partir de ahora nos centraremos en analizar dicho máster. En él se establecen unos objetivos (descritos como competencias que adquirir) que están en gran parte íntimamente relacionados con la Didáctica de las Ciencias Experimentales (y el resto de las didácticas específicas).

Dentro de la estructura del máster se contempla un bloque específico con un mínimo de 24 créditos relacionado, en nuestro caso, con la Didáctica de las Ciencias Experimentales (tabla 1). Dicho bloque se distribuye en tres materias.

\section{PROCEDIMIENTO SEGUIDO}

Partiendo de este marco legal nos planteamos cómo seleccionar las competencias específicas para el profesorado de secundaria en ciencias experimentales. Para ello era necesario, en primer lugar, ampliar y fundamentar las competencias prescritas en el máster, buscando integrar otros puntos de vista que enriquecieran el nuevo modelo de formación inicial del profesorado en España. Es lo que mostraremos en este trabajo. 
Tabla 1.

Bloque específico de contenidos de Didáctica de las Ciencias Experimentales en el Máster Universitario en Profesorado (las numeraciones de las competencias son nuestras)

\begin{tabular}{|c|c|}
\hline MATERIAS & COMPETENCIAS QUE DEBEN ADQUIRIRSE \\
\hline $\begin{array}{l}\text { Complementos para la } \\
\text { formación disciplinar }\end{array}$ & $\begin{array}{l}\text { 1. Conocer el valor formativo y cultural de las materias correspondientes a la especialización y los } \\
\text { contenidos que se cursan en las respectivas enseñanzas. } \\
\text { 2. Conocer la historia y los desarrollos recientes de las materias y sus perspectivas para poder } \\
\text { transmitir una visión dinámica de estas. } \\
\text { 3. Conocer contextos y situaciones en que se usan o aplican los diversos contenidos curriculares. }\end{array}$ \\
\hline $\begin{array}{l}\text { Aprendizaje y } \\
\text { enseñanza de } \\
\text { las materias } \\
\text { correspondientes }\end{array}$ & $\begin{array}{l}\text { 4. Conocer los desarrollos teórico-prácticos de la enseñanza y el aprendizaje de las materias co- } \\
\text { rrespondientes. } \\
\text { 5. Transformar los currículos en programas de actividades y de trabajo. } \\
\text { 6. Adquirir criterios de selección y elaboración de materiales educativos. } \\
\text { 7. Fomentar un clima que facilite el aprendizaje y ponga en valor las aportaciones de los alumnos. } \\
\text { 8. Integrar la formación en comunicación audiovisual y multimedia en el proceso de enseñanza- } \\
\text { aprendizaje. } \\
\text { 9. Conocer estrategias y técnicas de evaluación y entender la evaluación como un instrumento de } \\
\text { regulación y estímulo al esfuerzo. }\end{array}$ \\
\hline $\begin{array}{l}\text { Innovación docente } \\
\text { e iniciación a la } \\
\text { investigación educativa }\end{array}$ & $\begin{array}{l}\text { 10. Conocer y aplicar propuestas docentes innovadoras en el ámbito de la especialización cursada. } \\
\text { 11. Analizar críticamente el desempeño de la docencia, de las buenas prácticas y de la orientación } \\
\text { utilizando indicadores de calidad. } \\
\text { 12. Identificar los problemas relativos a la enseńanza y aprendizaje de las materias de la especiali- } \\
\text { zación y plantear alternativas y soluciones. } \\
\text { 13. Conocer y aplicar metodologías y técnicas básicas de investigación y evaluación. }\end{array}$ \\
\hline
\end{tabular}

Resulta obvio que la Didáctica de las Ciencias Experimentales puede realizar aportaciones al proceso de formación del profesorado de secundaria basadas en las investigaciones propias de su ámbito de trabajo, pero también es cierto que existen otras disciplinas y campos de estudio que son igualmente influyentes, por lo que enunciar un listado de competencias específicas para la formación del futuro profesorado de ciencias requiere un modelo transdisciplinar, en el que las diferentes visiones que emanan de las disciplinas deben integrarse. Por consiguiente, el marco teórico que puede guiar este proceso debe contemplar fuentes de origen diverso y llegar más lejos que los tradicionales enfoques interdisciplinares, en los que las distintas aportaciones no se integran en un proceso metodológico único.

Como antecedente más relevante en el que se debiera fundamentar y orientar nuestra investigación, se ha de destacar el EEES. Se trata del marco general donde se inscribe el máster que nos ocupa como título universitario de posgrado, definido además en este caso como un título "profesionalizador». Uno de los pilares en los que se apoya es en la necesidad de formar a los alumnos en competencias.

El perfil profesionalizador del máster debería implicar la adquisición de los grandes principios de la profesión docente por parte de los futuros profesores y que, en nuestro caso, proceden de la Didáctica General, la Didáctica de las Ciencias Experimentales y los requerimientos de la actual estructura de la educación no universitaria (competencias contempladas para los estudiantes en los currículos oficiales para la educación secundaria obligatoria y no obligatoria en España). Igualmente se debería tener en cuenta la opinión de profesores en activo en cuanto a la formación inicial en competencias que recibieron y las demandas reales que perciben en el ejercicio de su profesión.

Por otro lado, contemplamos las fuentes sociales, para las que, como mencionábamos al principio de este trabajo, deberíamos tener en cuenta las competencias evaluadas por el Programa PISA, así como las demandas sociales, tal y como se conciben hoy día a través de las tendencias curriculares «Alfabetización Científica» o "Ciencia-Tecnología-Sociedad (CTS)». 
En el próximo apartado profundizaremos en algunos aspectos de estas fuentes curriculares, representadas en la figura 1, y extraeremos de ellos las competencias que se deriven.

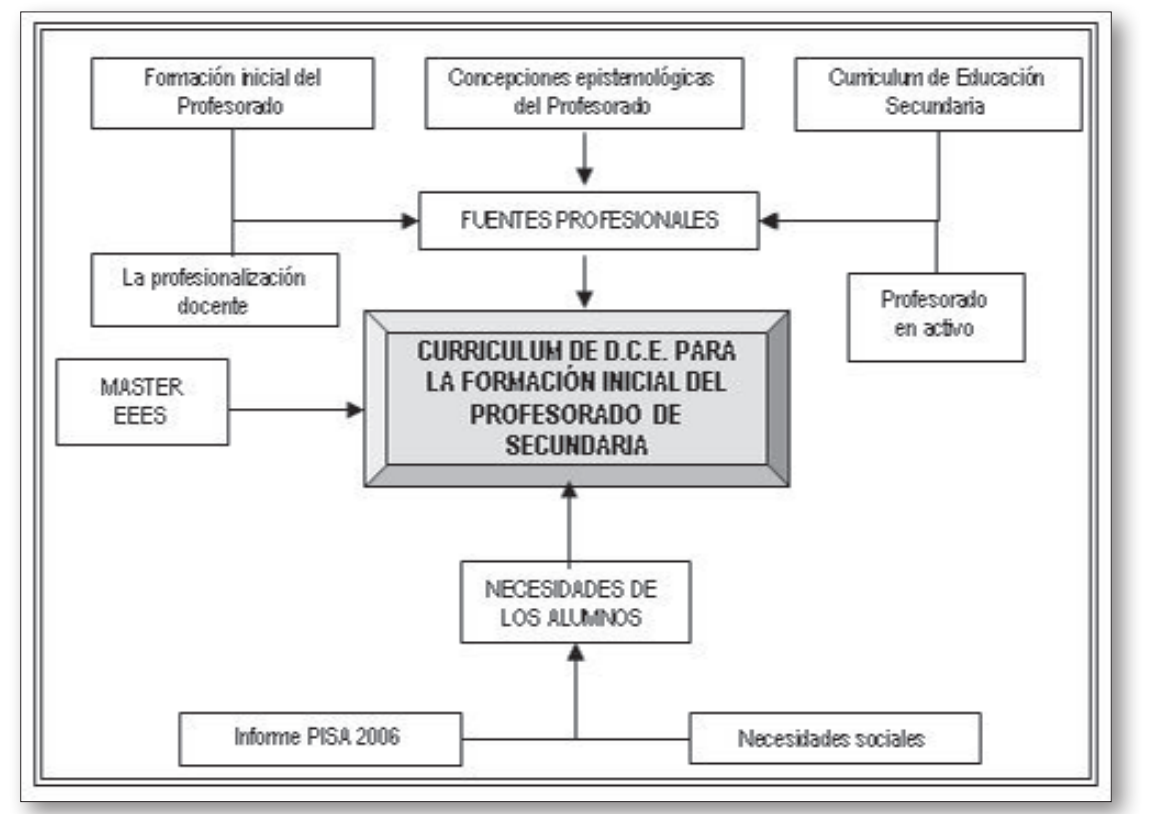

Fig 1. Fuentes curriculares consideradas

\section{FUENTES UTILIZADAS EN EL DISEÑO DEL CURRÍCULO DE LA FORMACIÓN INICIAL DEL PROFESORADO}

\section{Modelos de formación inicial del profesorado}

La formación inicial del profesorado constituye un tópico clásico en el ámbito de la Didáctica, compartido, con los matices correspondientes, también por la Didáctica de las Ciencias Experimentales. En ambos casos se puede llegar a enunciar líneas de actuación que pasan por la superación de un modelo en el que el conocimiento científico adquirido en la licenciatura -creencias del profesorado- (Roehrig, Kruse y Kern, 2007) resulta claramente insuficiente para tomar decisiones sobre qué enseñar y cómo hacerlo (Valcárcel y Sánchez, 2000), pues la ausencia de formación didáctica repercute negativamente en el propio conocimiento del contenido (Mellado y González, 2000) y en la forma de enseñar posterior más que cualquier capacitación didáctica (Porlán, Rivero y Martín, 1998). Los distintos modelos que han surgido en los últimos años consideran especialmente la influencia de este componente. Otro elemento de los programas de formación del profesorado es el conjunto de creencias que el futuro docente tiene sobre su capacidad (las creencias de autoeficacia) (Bandura, 1997; Abell 2007). En general, sobresalen los modelos que poseen un enfoque constructivista (Posnanski, 2002) y los que se centran en la enseńanza a través de la indagación (Singer et al., 2011). En este sentido, como alternativas al modelo tradicional, se han desarrollado o planteado algunas recomendaciones:

- Establecer grados específicos de profesorado en ciencias o un itinerario formativo-docente en el seno de los grados de ciencias.

- Apostar por una formación científica del futuro profesor menos especializada y más volcada hacia una visión global de la ciencia. Al mismo tiempo habría que prestar atención a las materias 
que forman su entorno, como la historia, la epistemología y las implicaciones ciencia-tecnología-sociedad-ambiente.

- El currículo de la formación didáctica del futuro profesor de secundaria debería contemplar igualmente conocimientos sobre psicología del aprendizaje, pedagogía y sociología de la educación.

- La metodología de la formación inicial debería diseñarse en relación con problemas prácticos. Debe permitirse un análisis teórico, un diseño de alternativas, una experimentación y una evaluación. Tampoco deben descuidarse las exigencias metodológicas y organizativas que plantea el uso de recursos extraescolares.

- El periodo de prácticas debería articularse con la teoría (Luft, 2007) y, además, favorecer la conexión alumno-centro escolar-facultad. El intercambio entre los distintos niveles educativos contribuiría a enriquecer la formación de los futuros profesores.

En el caso del máster que estamos analizando está claro que algunas de estas recomendaciones pueden ser contempladas en las competencias que establezcamos, pero no sucede así con la primera de ellas, dado que, como ya hemos indicado, la opción adoptada en España para la formación inicial ha sido la de un complemento de la formación en ciencias recibida previamente.

A continuación veremos cómo la intervención de distintas fuentes para la definición de las competencias deseables permitirá dar cabida a las alternativas anteriores.

\section{El currículo de ciencias en la educación secundaria obligatoria}

Las Enseñanzas Mínimas de la Educación Secundaria Obligatoria (Ministerio de Educación y Ciencia, 2007b) definen las siguientes competencias que los alumnos de esta etapa educativa habrían de adquirir, directamente relacionadas con nuestros intereses (las numeraciones son nuestras):

1. Competencia en el conocimiento y la interacción con el mundo físico. Habilidad para interactuar con el mundo físico, tanto en sus aspectos naturales como en los generados por la acción humana.

2. Tratamiento de la información y competencia digital. Disponer de habilidades para buscar, obtener, procesar y comunicar información, y para transformarla en conocimiento.

3. Competencia para aprender a aprender. Disponer de habilidades para iniciarse en el aprendizaje y ser capaz de continuar aprendiendo de manera cada vez más eficaz y autónoma de acuerdo con los propios objetivos y necesidades.

4. Autonomía e iniciativa personal. Adquisición de la conciencia y aplicación de un conjunto de valores y actitudes. Capacidad de demorar la necesidad de satisfacción inmediata, de aprender de los errores $y$ de asumir riesgos, de elegir con criterio propio, de imaginar proyectos, y de llevarlos adelante.

Posteriormente estas competencias se desarrollan más extensamente cuando se regulan las enseñanzas mínimas en cada materia de esta etapa educativa. Dado que la competencia más íntimamente relacionada con el conocimiento científico es la 1, identificaremos las coincidencias entre los descriptores de esta competencia y las competencias contempladas en la tabla 1, matizando estas últimas para lograr su integración.

\section{El Programa PISA}

Siguiendo con el propósito de este trabajo, ampliaremos la comparación al ámbito del Informe PISA (2006), dado que fue esta edición la última prueba con énfasis en ciencias. Para ello, buscaremos indicadores de las competencias que los autores de dicho informe asumen que los alumnos deben poseer y 
pretenden evaluar. Tomando como punto de partida el concepto de competencia científica individual y su evaluación en PISA, hemos elaborado la tabla 2.

Tabla 2.

Indicadores sobre competencia científica y su evaluación

presentes en PISA (2006) (la denominación de los indicadores A, B, C, etc. es nuestra)

Capacidad de los estudiantes a la hora de:

A. Identificar cuestiones científicas.

B. Explicar fenómenos científicamente.

C. Utilizar pruebas científicas.

El concepto de competencia científica aplicado a un individuo concreto hace referencia a los siguientes aspectos:

D. Conocimiento científico y el uso que se hace de ese conocimiento para identificar cuestiones, adquirir nuevos conocimientos, explicar fenómenos científicos y extraer conclusiones basadas en pruebas sobre temas relacionados con las ciencias.

E. La comprensión de los rasgos característicos de la ciencia, entendida como una forma del conocimiento y la investigación humanos.

F. La conciencia de las formas en que la ciencia y la tecnología moldean nuestro entorno material, intelectual y cultural. G. La disposición a implicarse en asuntos relacionados con la ciencia y a comprometerse con las ideas de la ciencia como un ciudadano reflexivo.

A efectos de esta evaluación, la definición de competencia científica de PISA 2006 puede caracterizarse por cuatro aspectos interrelacionados:

H. Contexto: reconocer las situaciones de la vida dotadas de un contenido científico y tecnológico.

I. Conocimientos: comprender el mundo natural por medio del conocimiento científico, en el que se incluye tanto el conocimiento del mundo natural como el conocimiento acerca de la propia ciencia.

J. Capacidades: acreditar que se poseen una serie de capacidades, como identificar cuestiones científicas, explicar fenómenos científicamente y extraer conclusiones basadas en pruebas.

K. Actitudes: mostrar interés por la ciencia, respaldar la investigación científica y contar con la motivación necesaria para actuar de forma responsable en relación, por ejemplo, con los recursos naturales y los ambientes.

Si comparamos los indicadores de la tabla 2 con las competencias de la tabla 1, encontramos que se hallan todos contemplados, excepto el indicador K parcialmente, en concreto: «mostrar interés por la ciencia, respaldar la investigación científica», por lo que habría que añadirlo en el nuevo listado.

\section{La opinión del profesorado en activo}

El tercer paso que pretendemos dar nos obliga a recoger la opinión del profesorado de educación secundaria en activo a partir de los siguientes interrogantes iniciales:

- ¿Qué competencias didácticas y profesionales deberían haber adquirido en su formación inicial?

- ¿Qué competencias científicas (incluyendo el conocimiento cotidiano y el axiológico) consideran que deberían adquirir sus alumnos?

- ¿Qué competencias científicas trabajan explícitamente con sus alumnos?

Para ello se elaboró un cuestionario en línea presentado como una escala tipo Likert que opera con una escala de opinión acuerdo-desacuerdo de 0 a 4, siendo 0 ninguna adquisición o ninguna frecuencia de uso (según sea el caso) y 4 lo contrario. Incluimos las competencias que hasta entonces habíamos seleccionado pero redactadas con el objetivo de tratar de expresar acciones comunes en la práctica de la 
enseñanza para que los profesores pudieran identificarse con los distintos ítems. Hubo varias versiones del cuestionario hasta su forma final (disponible en >http://www.ugr.es/-cudice/>), que fue validada por los miembros del equipo de investigación (12 profesores universitarios y de secundaria). También se consideró importante incluir un ítem sobre el uso de tecnologías de la información y la comunicación, dada su creciente importancia en las aulas actuales. El cuestionario contenía preguntas acerca de su formación inicial y, posteriormente, sobre las mismas competencias en su actividad actual como docentes. Se distribuyó a través del correo electrónico a todos los centros de educación secundaria de Andalucía $(\mathrm{N}=1021)$ y recibimos un total de 103 respuestas válidas.

En relación con el análisis de las respuestas es interesante centrarse en la diferencia entre las medias de «adquisición de competencias en la formación previa» y las de "frecuencia de uso», ya que representan la cercanía (o distancia) entre la formación recibida y lo que realmente se necesita para la enseñanza. Las medias y los niveles de significación de estas diferencias se muestran en la tabla 3.

Tabla 3.

Medias y nivel de significación de la diferencia

de medias entre la frecuencia de uso actual de las competencias señaladas y su adquisición en la formación inicial por el profesorado encuestado (n.s. no significativo; ${ }^{*} \mathrm{p}<0,05 ;{ }^{* *} \mathrm{p}<0,01$ ).

\begin{tabular}{|c|c|c|c|c|}
\hline Competencias & Acciones & $\begin{array}{l}\text { Media } \\
\text { adquisición } \\
\text { formación } \\
\text { inicial }\end{array}$ & $\begin{array}{c}\text { Media } \\
\text { frecuencia de } \\
\text { uso actual }\end{array}$ & $\begin{array}{c}\text { Nivel de } \\
\text { significación de } \\
\text { la diferencia } \\
\text { de medias }\end{array}$ \\
\hline \multirow{3}{*}{$\begin{array}{c}\text { I. } \\
\text { Aplicar el } \\
\text { conocimiento } \\
\text { científico a la } \\
\text { vida cotidiana: }\end{array}$} & 1. Para introducir un tema y motivar al alumnado & 2,5 & 4,2 & ** \\
\hline & 2. Durante el desarrollo del tema & 2,8 & 4,0 & ** \\
\hline & 3. Como actividades de ampliación del tema & 2,8 & 3,7 & $* *$ \\
\hline \multirow{4}{*}{$\begin{array}{c}\text { II. } \\
\text { Realizar } \\
\text { actividades de } \\
\text { lápiz y papel } \\
\text { para: }\end{array}$} & 1. Interpretar datos científicos y sacar conclusiones & 3,6 & 3,9 & $*$ \\
\hline & $\begin{array}{l}\text { 2. Resolver situaciones problemáticas que necesiten } \\
\text { de una investigación científica }\end{array}$ & 3,2 & 3,3 & n.s. \\
\hline & $\begin{array}{l}\text { 3. Reconocer hechos y datos propios de la } \\
\text { disciplina }\end{array}$ & 3,6 & 3,9 & n.s. \\
\hline & $\begin{array}{l}\text { 4. Reconocer y utilizar conceptos y teorías propios } \\
\text { de la disciplina }\end{array}$ & 3,7 & 4,1 & * \\
\hline \multirow{4}{*}{$\begin{array}{c}\text { III. } \\
\text { Mostrar ejemplos } \\
\text { para: }\end{array}$} & 1. Interpretar datos científicos y sacar conclusiones & 3,6 & 4,1 & ** \\
\hline & $\begin{array}{l}\text { 2. Resolver situaciones problemáticas que necesiten } \\
\text { una investigación científica }\end{array}$ & 3,3 & 3,6 & * \\
\hline & $\begin{array}{l}\text { 3. Reconocer hechos y datos propios de la } \\
\text { disciplina }\end{array}$ & 3,6 & 4,0 & * \\
\hline & $\begin{array}{l}\text { 4. Reconocer y utilizar conceptos y teorías propios } \\
\text { de la disciplina }\end{array}$ & 3,7 & 4,1 & ** \\
\hline \multirow{4}{*}{$\begin{array}{c}\text { IV. } \\
\text { Realizar } \\
\text { actividades de } \\
\text { laboratorio cuyo } \\
\text { objetivo es: }\end{array}$} & 1. Interpretar datos científicos y sacar conclusiones & 3,4 & 3,3 & n.s. \\
\hline & $\begin{array}{l}\text { 2. Resolver situaciones problemáticas que necesiten } \\
\text { una investigación científica }\end{array}$ & 3,1 & 3,0 & n.s. \\
\hline & $\begin{array}{l}\text { 3. Reconocer hechos y datos propios de la } \\
\text { disciplina }\end{array}$ & 3,4 & 3,3 & n.s. \\
\hline & $\begin{array}{l}\text { 4. Reconocer y utilizar conceptos y teorías propios } \\
\text { de la disciplina }\end{array}$ & 3,3 & 3,3 & n.s. \\
\hline
\end{tabular}




\begin{tabular}{|c|c|c|c|c|}
\hline Competencias & Acciones & $\begin{array}{c}\text { Media } \\
\text { adquisición } \\
\text { formación } \\
\text { inicial } \\
\end{array}$ & $\begin{array}{l}\text { Media } \\
\text { frecuencia de } \\
\text { uso actual }\end{array}$ & $\begin{array}{l}\text { Nivel de } \\
\text { significación de } \\
\text { la diferencia } \\
\text { de medias }\end{array}$ \\
\hline \multirow{3}{*}{$\begin{array}{c}\text { V. } \\
\text { Realizar prácticas } \\
\text { de campo cuyo } \\
\text { objetivo es: }\end{array}$} & 1. Reconocer hechos propios de la disciplina & 2,9 & 2,7 & n.s. \\
\hline & $\begin{array}{l}\text { 2. Obtener datos, interpretarlos y sacar } \\
\text { conclusiones }\end{array}$ & 2,9 & 2,7 & n.s. \\
\hline & 3. Sacar conclusiones a partir de la observación & 2,9 & 2,7 & n.s. \\
\hline \multirow{2}{*}{$\begin{array}{c}\text { VI. } \\
\text { Analizar } \\
\text { y discutir } \\
\text { diversos tipos } \\
\text { de documentos } \\
\text { para: }\end{array}$} & $\begin{array}{l}\text { 1. Identificar las implicaciones éticas resultantes de } \\
\text { la actividad científica }\end{array}$ & 2,5 & 3,4 & ** \\
\hline & $\begin{array}{l}\text { 2. Reconocer la responsabilidad de la actividad } \\
\text { científica con el desarrollo sostenible }\end{array}$ & 2,6 & 3,7 & ** \\
\hline \multirow{2}{*}{$\begin{array}{c}\text { VII. } \\
\text { Desarrollar } \\
\text { actividades de } \\
\text { evaluación para: }\end{array}$} & 1. Favorecer y estimular los procesos de aprendizaje & 2,5 & 4,1 & ** \\
\hline & $\begin{array}{l}\text { 2. Regular y controlar las actividades de aula de los } \\
\text { estudiantes }\end{array}$ & 2,5 & 4,2 & $* *$ \\
\hline \multirow{4}{*}{$\begin{array}{c}\text { VIII. } \\
\text { Utilizar los } \\
\text { recursos que } \\
\text { brindan las } \\
\text { tecnologías de la } \\
\text { información y de } \\
\text { la comunicación } \\
\text { para: }\end{array}$} & $\begin{array}{l}\text { 1. Manejar recursos básicos de ofimática (editores } \\
\text { de texto, bases de datos, gráficos, etc.) para } \\
\text { tareas educativas y administrativas }\end{array}$ & 2,2 & 4,2 & ** \\
\hline & $\begin{array}{l}\text { 2. Realizar presentaciones de carácter didáctico con } \\
\text { el uso del software correspondiente }\end{array}$ & 2,0 & 3,8 & ** \\
\hline & $\begin{array}{l}\text { 3. Acceder a internet para obtener información } \\
\text { sobre contenidos, recursos didácticos en general, } \\
\text { poder comunicarse con otros profesores, etc. }\end{array}$ & 2,2 & 4,3 & ** \\
\hline & $\begin{array}{l}\text { 4. Utilizar recursos multimedia de la red para } \\
\text { simulaciones que facilitan la enseńanza de un } \\
\text { contenido determinado }\end{array}$ & 2,0 & 3,8 & ** \\
\hline \multirow{2}{*}{$\begin{array}{c}\text { IX. } \\
\text { Usar la } \\
\text { innovación e } \\
\text { investigación } \\
\text { educativas para: }\end{array}$} & $\begin{array}{l}\text { 1. Reconocer los problemas en la enseńanza y } \\
\text { aprendizaje de la especialidad }\end{array}$ & 2,2 & 3,5 & $* *$ \\
\hline & $\begin{array}{l}\text { 2. Realizar propuestas innovadoras en la docencia } \\
\text { de la especialidad que planteen soluciones a los } \\
\text { problemas }\end{array}$ & 2,1 & 3,4 & $* *$ \\
\hline
\end{tabular}

Se observa que la mayoría de las diferencias son significativas. Por establecer un criterio exigente, vamos a considerar solo aquellas competencias cuya diferencia de medias entre el uso que se hace de ellas en el aula y la formación inicial recibida sea mayor que 1; esto sucede con las competencias relacionadas con:

- Aplicar el conocimiento científico a la vida cotidiana, para introducir un tema y motivar al alumnado (competencia I.1), y durante el desarrollo del tema (competencia I.2).

- Analizar y discutir diversos tipos de documentos (competencia VI, en todos sus apartados).

- Desarrollar actividades de evaluación (competencia VII, en todos sus apartados).

- Utilizar los recursos que brindan las tecnologías de la información y de la comunicación (competencia VIII, en todos sus apartados).

- Usar la innovación e investigación educativa (competencia IX, en sus dos apartados). 
Cabe destacar que todas estas competencias no tenían presencia o era muy escasa en los modelos de enseñanza anterior (modelo del CAP), como las competencias VIII y IX, o se refieren a temas que suelen presentar más dificultades para los profesores (competencias I y VII).

En las competencias II (Realizar actividades de lápiz y papel) y III (Mostrar ejemplos), la diferencia entre la frecuencia de uso en el aula y la formación previa también es positiva, lo que indica que se necesitaría más formación de la recibida, aunque en menor medida que en las señaladas en el párrafo anterior.

El uso en el aula de las competencias IV (Realizar actividades de laboratorio) y V (Realizar prácticas de campo) queda cubierto con la formación recibida. En algunos casos incluso aquella supera la frecuencia de uso en el aula.

En definitiva, al menos deberíamos integrar las competencias con mayor déficit (diferencia de medias mayor que 1) en la tabla 1 . A primera vista, la integración parece factible modificando o matizando algunas de las competencias presentes en aquella.

\section{Concepciones epistemológicas del profesorado}

Continuando con nuestras fuentes para el diseño del currículo de la formación inicial del profesorado, abordamos una revisión bibliográfica sobre las concepciones epistemológicas del profesorado. Ello se hizo habida cuenta de que dichas concepciones condicionan, en gran medida, no solo la visión de la ciencia que los profesores poseen, sino la que transmiten en su enseńanza.

Si la Naturaleza de la Ciencia $(\mathrm{NdC})$ se va a incluir en el currículo, como se propone en los actuales enfoques para la enseñanza de ciencias, es esencial capacitar a los profesores para ello. Estos disponen de ideas preconcebidas muy arraigadas que deben ser investigadas. El denominador común siempre es una visión ingenua de la ciencia, marcada por una fuerte dosis de empirismo e inductivismo. Así, por ejemplo, McComas (1998) describe quince visiones incorrectas de la NdC. Estos «mitos de la ciencia», como él los denomina, son ideas mantenidas por muchos profesores de ciencias y que se pueden encontrar también en los libros de texto.

Parece haberse concluido que como mejor puede aprenderse la $\mathrm{NdC}$ es a través de la enseñanza explícita y reflexiva en diferentes contextos de aprendizaje, más que implícitamente a través de actividades basadas en procedimientos científicos, pero aún se sabe muy poco sobre cómo modificar eficazmente las creencias de los profesores (Acevedo, 2008). Aunque algunas parecen evolucionar adecuadamente, en general el conocimiento adquirido durante los programas de formación no es muy sólido y suele desaparecer con el tiempo. Las mejores propuestas de enseñanza parecen estar basadas en una estructura que consta de ciclos de instrucción, enseñanza práctica y reflexión (Lotter et al., 2009).

En cuanto a qué contenido de $\mathrm{NdC}$ se debe enseñar, aunque hay cierto desacuerdo en este sentido, no es difícil llegar a un consenso sobre determinados aspectos que deben ser incluidos (Acevedo et al., 2007). A este respecto, Osborne et al. (2003) han elaborado una lista de ideas clave tomadas de las opiniones de más de una veintena de expertos y la aplicación de criterios de consenso muy estrictas (> $2 / 3$ de expertos). Los temas propuestos son:

a) Ciencia y curiosidad. Creatividad. Hipótesis y predicción.

b) Métodos científicos y comprobación crítica.

c) Análisis e interpretación de datos.

d) Diversidad del pensamiento científico.

e) Ciencia y certeza.

f) Desarrollo histórico del conocimiento científico.

g) Dimensiones morales y éticas del desarrollo del conocimiento científico.

h) Cooperación y colaboración en el desarrollo del conocimiento científico. 
Si revisamos qué aspectos de los mencionados por Osborne et al. (2003) son contemplados como competencias en la selección que llevamos realizada, observamos cómo solo lo están los $f$ y $g$. Por tanto, sería conveniente incorporar el resto de los puntos como subcompetencias de la 2 (tabla 1).

\section{La profesionalización docente}

Retomando el concepto de competencia profesional que abordamos al principio de este trabajo, decíamos que, por lo general, el desarrollo de competencias obedece a la definición del perfil profesional y de las tareas y funciones de este. En el caso del profesor de educación secundaria se pueden identificar cuatro funciones:

a) Planificar e implementar el currículo escolar.

b) Tutorizar a los alumnos.

c) Apoyarse en el contexto familiar y social.

d) Actualizarse e implicarse en la profesión docente.

La $b$ y la $c$ pueden considerarse genéricas o equivalentes para los diversos especialistas, mientras que la $a$ y la $d$ entran de lleno en el ámbito de las didácticas específicas. A partir de tales funciones, que no son solo docentes, el desarrollo de competencias se puede planificar a partir de tres enfoques:

1. Competencias a través de las tareas profesionales desempeñadas.

2. Competencias a través de atributos personales.

3. Competencias desde una perspectiva holística.

Vamos a asumir el tercer enfoque, que integra los dos anteriores. Entre los modelos de competencias basados en dicha perspectiva, adoptamos el modelo de Echevarría (2001, 2004), pues ha sido utilizado en el Proyecto Tuning y el Máster de Profesor de Secundaria es una titulación de posgrado claramente profesionalizante y orientada a la acción.

La competencia para la acción profesional, como conjunto de saberes integrados, se estructura desde cuatro vértices:

- Competencias técnicas (saber): poseer conocimientos especializados y relacionados con determinado ámbito profesional, que permiten dominar como experto los contenidos y las tareas acordes a su actividad laboral.

- Competencias metodológicas (saber hacer): saber aplicar los conocimientos a situaciones laborales concretas, utilizar procedimientos adecuados a las tareas pertinentes, solucionar problemas de manera autónoma y transferir con ingenio las experiencias adquiridas a situaciones novedosas.

- Competencias participativas (saber estar): estar atento a la evolución del mercado laboral, predispuesto al entendimiento interpersonal, dispuesto a la comunicación y cooperación con los demás y demostrar un comportamiento orientado hacia el grupo.

- Competencias personales (saber ser): tener una imagen realista de sí mismo, actuar conforme a las propias convicciones, asumir responsabilidades, tomar decisiones y relativizar las posibles frustraciones.

De un análisis de las competencias seleccionadas hasta ahora, se deduce una visión de la formación centrada en el saber y el saber hacer, en donde dominan los elementos disciplinares y metodológicos. No obstante, en el desempeño profesional existen otras competencias complementarias con un carácter interdisciplinar, tales como: 
- Las competencias lingüísticas, que resulta insuficiente reducirlas a la expresión oral o a la comprensión de textos científicos. Pueden interpretarse igualmente desde el punto de vista global como la adquisición de los lenguajes de la ciencia (multimodales).

- Forma parte de la competencia matemática la habilidad para interpretar y expresar con claridad y precisión informaciones, datos y argumentaciones, pero cobra sentido cuando es utilizada para enfrentarse a la observación e interpretación de fenómenos naturales y de la vida cotidiana, lo cual se realizará en las clases de Ciencias Experimentales.

- Las competencias social y ciudadana están directamente relacionadas con actitudes, valores y el desarrollo ético. A este efecto deberían considerarse los valores contextuales con los que abordar el tratamiento de dilemas y controversias públicas sociotecnológicas.

Este último análisis nos obligará a reelaborar el listado de competencias disponible, incorporando las que se derivan del análisis de un modelo de formación basado en competencias para la acción profesional. Para ello, en algunos casos se han matizado y diferenciado algunas competencias ya seleccionadas, al considerar que se trata de competencias genéricas, como el compromiso ético que en un posgrado deben darse por conseguidas, pero hemos introducido también especificaciones sobre la realidad multicultural de los centros educativos, que también merecen su tratamiento en las clases de ciencias (Cabo y Enrique, 2004) y una competencia relacionada con la realidad de aula, esencial para el futuro desarrollo profesional (Schwarz, 2009). Por otro lado, el desarrollo sostenible se ha incorporado como eje integrador, con una perspectiva holística y considerando las interrelaciones existentes entre las distintas dimensiones de sostenibilidad. El tratamiento de dilemas, debates y controversias en el aula (véase el próximo apartado), o el planteamiento de problemas de la vida cotidiana debe hacerse analizando problemas complejos en los que intervienen muy diversos factores, que no pueden tratarse exclusivamente por separado por el carácter sistémico de las interrelaciones.

\section{Necesidades sociales}

En este último apartado queremos referirnos al protagonismo de la ciencia en los medios de comunicación, en tanto referente social más próximo de los estudiantes de ciencia y de los futuros profesores, actuando como mediadores entre la cultura científica global y la personal de los ciudadanos (BaramTsabari y Yarden, 2005). Incluso, como han evidenciado Halkia y Mantzouridis (2005), los artículos sobre ciencia en la prensa escrita son vistos por los estudiantes de educación secundaria como más interesantes y comprensibles que los libros de texto de ciencia. Desde el punto de vista de la enseńanza de las ciencias, los medios de comunicación toman protagonismo en relación con la denominada perspectiva CTS, sobre todo con la importancia que adquiere el concepto de ciencia para todos y el de la alfabetización científico-tecnológica, que se consolida como la finalidad principal de la enseñanza científico-tecnológica en las etapas obligatorias, frente a la formación de futuros científicos y tecnólogos (Membiela, 2001). El concepto de alfabetización científico-tecnológica tiene su origen precisamente en la capacidad de los ciudadanos para leer y comprender noticias científicas y tomar decisiones sobre ellas, aunque no existe un consenso total en cuanto a su significado.

Diversas encuestas realizadas en España por la Fundación Española de Ciencia y Tecnología (FECYT) evidencian la existencia de una demanda de información por parte de los ciudadanos que se manifiesta en un alto grado de interés hacia las noticias científicas, si bien al compararse con el grado de conocimiento aporta un balance negativo, es decir, el interés es mayor que el conocimiento, aunque menor que hacia los temas deportivos, por ejemplo. Hay que destacar también que las respuestas hacia la ciencia y la tecnología en general son peores que para medicina y salud, alimentación y consumo, o medio ambiente y ecología. Según la encuesta de la FECYT del ańo 2008, los medios para informarse 
sobre ciencia y tecnología con mayor penetración son la televisión en primer lugar (59,9\%) y la prensa escrita en segundo $(33,8 \%)$, considerándose insuficiente la información sobre ciencia y tecnología en los medios en general. Temas controvertidos como los cultivos transgénicos, el dopaje en el deporte, las antenas de telefonía móvil, la energía nuclear, etc. constituyen excelentes oportunidades para el trabajo con estudiantes de educación secundaria (Kolstø, 2001).

El actual Máster de Profesorado debería responder a la necesidad general de formar a futuros ciudadanos (estudiantes de educación secundaria) que habrán de tomar decisiones individuales y colectivas sobre ciencia y tecnología. Además, la rápida evolución del conocimiento científico y tecnológico requiere de ellos una formación para toda la vida, en la que los medios de comunicación se convierten en mediadores privilegiados entre la ciencia-tecnología y la sociedad. Por ello, la futura formación de profesionales de la educación científica debería atender a las siguientes cuestiones:

- Aportar información sobre la comunicación social de la ciencia y la tecnología.

- Ayudar a la construcción de una visión compleja de las relaciones CTS, contribuyendo a un concepto de ciencia y tecnología como procesos sociales y considerando la cultura científica como parte indisoluble de la cultura humana.

- Desarrollar la capacidad para relacionar datos científicos y tecnológicos con otros ámbitos de conocimiento, como los de tipo social, político, económico y ético, en condiciones de razonamiento no formal, en decir, en contexto.

- Desarrollar la capacidad para utilizar los medios de comunicación como recurso educativo en actividades de aula, entrenando a los estudiantes en la lectura crítica y con criterios independientes de las noticias sobre ciencia y tecnología en dichos medios.

- El tratamiento de noticias sobre debates y controversias científicas y tecnológicas debe responder a criterios didácticos como su actualidad y el contexto local del aula, por lo que no es posible establecer criterios universales sobre el tipo de noticias que tratar.

- Desde el punto de vista de las noticias que despiertan mayor interés en la ciudadanía, las cuestiones aplicadas o de incidencia directa en la vida de las personas (la medicina y salud, el medio ambiente y la ecología, y la alimentación y consumo), prevalecen sobre las temáticas de ciencia y tecnología en general.

Estas recomendaciones deberían ser incorporadas a nuestro último listado de competencias, tal y como hemos hecho con anterioridad, mediante inclusiones de nuevas competencias o complementando las anteriores. Es lo que hemos hecho en la tabla 4, en la que se presenta nuestra propuesta final de competencias.

\section{CONCLUSIONES}

En este artículo hemos querido poner de manifiesto que, a partir de una reforma educativa institucional, es posible y deseable plantearse la formación inicial del profesorado desde una perspectiva ambiciosa y holística. La enseñanza de las ciencias en un mundo tan cambiante como el que nos toca vivir precisa no solo traspasar la frontera de la ciencia como contenido académico, sino incorporar otras perspectivas disciplinares, sociales, tecnológicas, históricas, éticas y, cómo no, también psicodidácticas. Ello debería conducir a ver la formación inicial del profesorado como una oportunidad para preparar a los futuros profesores para afrontar el reto de ser, además de profesores de ciencias, educadores en un sentido integral. Esto lo hemos traducido en una selección de competencias que podrían actuar como catalizadores de esta visión amplia de las fuentes curriculares. 
La investigación educativa en aspectos propios de la Didáctica de las Ciencias Experimentales, como las concepciones epistemológicas del profesorado, el currículo de ciencias de los alumnos de educación secundaria, el Programa internacional de evaluación PISA, o la propia ciencia informal, nos han proporcionado referencias en las que apoyarnos, pero también lo han hecho las perspectivas didácticas generales, como los modelos de formación inicial del profesorado, la profesión docente o la innovación e investigación educativas.

Bien es cierto que esto solo representa un paso en lo que es la implementación de un auténtico proceso formativo novedoso y eficaz (Davis y Smithey, 2009), pero tampoco es menos cierto que se trata de un paso necesario fruto de una reflexión colectiva en cuanto a qué competencias debiera adquirir el futuro profesor de ciencias.

En cuanto a las competencias concretas deseables para el futuro profesor que han surgido del procedimiento seguido en este trabajo, y que se ańaden a o matizan las que se mencionaron en la tabla 1 , las sintetizaremos a continuación en función de las materias del máster en las que se ubicarían (tabla 4):

- Complementos para la formación disciplinar. Hemos incorporado una competencia nueva y diversas subcompetencias que enriquecen las previstas en la normativa reguladora del máster. En general, hacen referencia a: la consideración de la ciencia en un sentido amplio como herramienta para lograr una mayor calidad de vida, comprender mejor el mundo e incrementar el interés de los estudiantes; a los valores éticos que debiera conllevar su desarrollo en la sociedad; a diferenciarla de otras formas de conocimiento pero desempeñando un papel crucial en la cultura contemporánea; a reconocer la labor investigadora consustancial con la ciencia, tanto actual como históricamente, y los métodos que la regulan, así como a la necesaria capacitación de los estudiantes en procedimientos y actitudes básicos en el quehacer científico.

- Aprendizaje y enseñanza de las materias correspondientes. Hemos añadido varias subcompetencias a las ya contempladas en la normativa, que se refieren fundamentalmente a: el compromiso deseable en los alumnos hacia la sostenibilidad y la diversidad cultural; el fomento de su espíritu crítico frente a los mensajes de los mass media, y la necesidad de la incorporación de estos últimos en las actividades de aula y de una comunicación social de la ciencia.

- Innovación docente e iniciación a la investigación educativa. Hemos ampliado los conceptos de innovación e investigación educativas previstos en la normativa, unificando dos competencias y añadiendo otras tres, las cuales hacen referencia a: el papel de los conceptos mencionados como generadores de propuestas alternativas y de solución a los problemas relacionados con la enseñanza y aprendizaje de las ciencias; el análisis crítico de la docencia habitual teniendo como referentes las buenas prácticas y los indicadores de calidad de esta, implicándose en tareas de renovación y actualización; concebir la innovación y la investigación como acciones que debieran implicar al resto de las disciplinas educativas del centro, y desarrollar las competencias inherentes a estas en el contexto del prácticum del máster. 
Tabla 4.

Propuesta final de competencias y subcompetencias

del futuro profesor de ciencias de educación secundaria

\begin{tabular}{|c|c|}
\hline ÁMBITO & COMPETENCIA \\
\hline $\begin{array}{l}\text { Complementos } \\
\text { para la forma- } \\
\text { ción disciplinar }\end{array}$ & 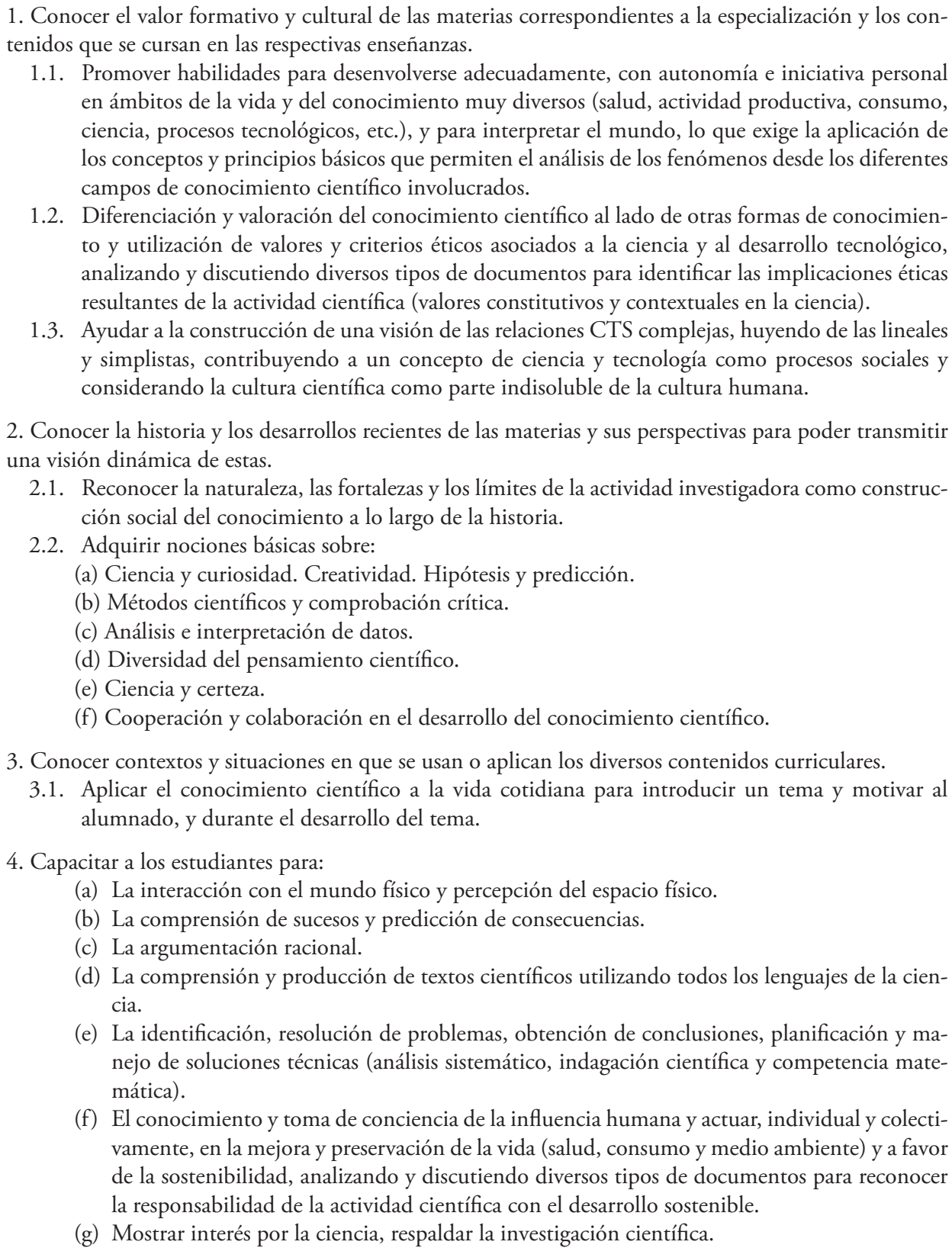 \\
\hline
\end{tabular}




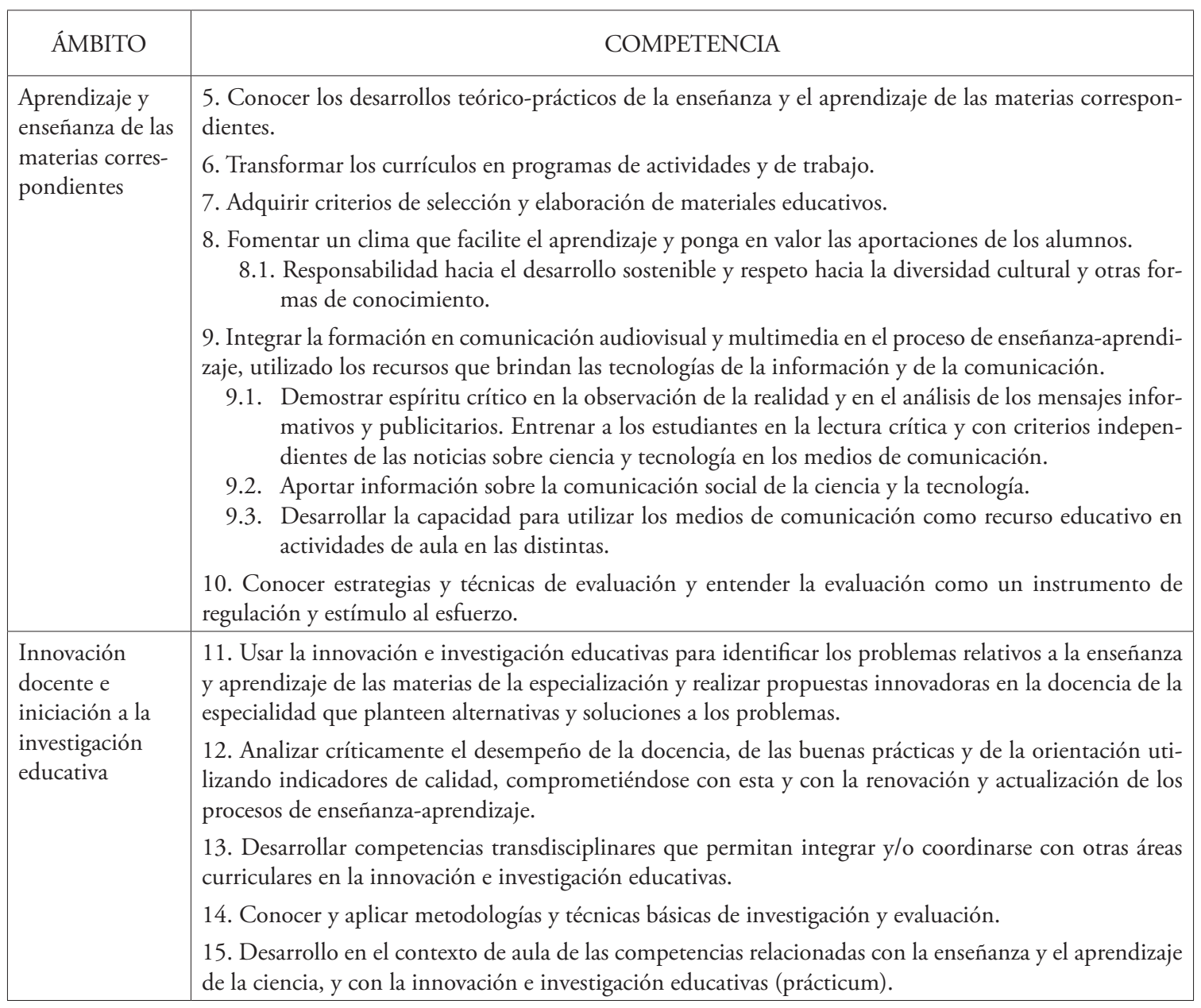

\section{AGRADECIMIENTOS}

Este artículo se inscribe en el Proyecto en vigor: EDU2008-02059 del Plan Nacional de I+D+I 2008 del Ministerio de Ciencia e Innovación (<http://www.ugr.es/ - cudice/>).

\section{BIBLIOGRAFÍA}

Abell, S. K. (2007). Research on science teacher knowledge, pp. 1105-1149. En: S. K. Abell y N. G. Lederman, (eds.). Handbook of Research on Science Education. Gran Bretańa: Routledge.

Acevedo, J. A. (2008). El estado actual de la naturaleza de la ciencia en la didáctica de las ciencias. Revista Eureka de Enseñanza y Divulgación de la Ciencia, 5(2), pp. 134-169.

Acevedo, J. A.; Vázquez, A.; Manassero, M. A. y Acevedo, P. (2007). Consensos sobre la naturaleza de la ciencia: Fundamentos de una investigación empírica. Revista Eureka de Enseñanza y Divulgación de la Ciencia, 4(1), pp. 42-66.

Bandura, A. (1997). Self-efficacy: The Exercise of Control. New York: Freeman.

Baram-Tsabari, A. y Yarden, A. (2005). Text genre as a factor in the formation of scientific literacy. Journal of Research in Science Teaching, 42(4), pp. 404-428.

http://dx.doi.org/10.1002/tea.20063 
Cabo, J. M. y Enrique, C. (2004). Hacia un concepto de Ciencia Intercultural. Enseñanza de las Ciencias, 22(1), pp. 137-146

Davis, E. A. y Smithey, J. (2009). Beginning Teachers Moving Toward Effective Elementary Science Teaching. Science Education, 93(4), pp. 745-770. http://dx.doi.org/10.1002/sce.20311

Echeverría, B. (2001). Gestión de la Competencia de Acción Profesional. Revista de Investigación Educativa, 20(1), pp. 7-43.

Echeverría, B. (2004). «Saber» y «sabor» de la profesionalidad. Herramientas, 74, pp. 6-11.

González, J. y WagenaAr, R. (eds.) (2003). Tuning Educational Structures in Europe. Informe final Fase 1. Bilbao: Universidad de Deusto.

Halkia, K. y Mantzouridis, D. (2005). Students' Views and Attitudes towards the Communication Code Used in Press Articles about Science. International Journal of Science Education, 27(12), pp. 1395-1411.

http://dx.doi.org/10.1002/sce.20311

Kaya, O. N. (2009). The Nature of Relationships among the Components of Pedagogical Content Knowledge of Preservice Science Teachers: «Ozone layer depletion» as an example. International Journal of Science Education, 31(7), pp. 961-988.

http://dx.doi.org/10.1080/09500690801911326

Kolstø, S. D. (2001). «To Trust or not to Trust...»- Pupils’ Ways of Judging Information Encountered in a Socio-Scientific Issue. International Journal of Science Education, 23(9), pp. 877-901 http://dx.doi.org/10.1080/09500690010016102

Lotter, C.; Singer, J. y Godley, J. (2009). The influence of Repeated Teaching and Reflection on Preservice Teachers' Views of Inquiry and Nature of Science. Journal of Science Teacher Education, 20(6), pp. 553-582.

http://dx.doi.org/10.1007/s10972-009-9144-9

Luft, J. (2007). Minding the Gap: Needed Research on Beginning/Newly Qualified Science Teachers. Journal of Research in Science Teaching, 44(4), pp. 532-537.

http://dx.doi.org/10.1002/tea.20190

Mccomas, W. F. (ed.) (1998). The Nature of Science in Science Education. Rationales and Strategies. Dordrecht: Kluwer.

Mellado, V. y González, T. (2000). La formación inicial del profesorado de ciencias, pp. 535-555. En: F. J. Perales y P. Cañal (eds.). Didáctica de las Ciencias Experimentales. Alcoy: Marfil.

Membiela, P. (2001). Enseñanza de las Ciencias desde la perspectiva Ciencia Tecnología Sociedad. Madrid: Narcea.

MINISTERIO DE EDUCACIÓN Y CIENCIA (2007a). Orden ECI/3858/2007, de 27 de diciembre, por la que se establecen los requisitos para la verificación de los títulos universitarios oficiales que habiliten para el ejercicio de las profesiones de Profesor de Educación Secundaria Obligatoria y Bachillerato, Formación Profesional y Enseñanzas de Idiomas, Boletín Oficial del Estado, 312, pp. 53751-53753.

MINISTERIO DE EDUCACIÓN Y CIENCIA (2007b). RD 1631/2006, de 29 de diciembre, por el que se establecen las enseñanzas mínimas correspondientes a la Educación Secundaria Obligatoria, Boletin Oficial del Estado, 5, pp. 667-773.

Osborne, J.; Collins, S.; Ratcliffe, M.; Millar, R. y Duschl, R. (2003). What «Ideas-aboutScience» Should Be Taught in School Science? A Delphi Study of the Expert Community. Journal of Research in Science Teaching, 40(7), pp. 692-720.

http://dx.doi.org/10.1002/tea.10105 
PISA (2006). Programa para la Evaluación Internacional de Alumnos de la OCDE. Informe Español. $<$ http://www.mec.es/multimedia/00005713.pdf> (consultado el 20 de noviembre de 2011).

Porlán, R.; Rivero, A. y Martín, R. (1998). Conocimiento profesional y epistemología de los profesores II: estudios empíricos y conclusiones. Enseñanza de las Ciencias, 16(2), pp. 271-289.

Posnanski, T. J. (2002). Professional Development Programs for Elementary Science Teachers: An Analysis of Teacher Self-Efficacy Beliefs and a Professional Development Model. Journal of Science Teacher Education, 13(2), pp. 189-220.

http://dx.doi.org/10.1023/A:1016517100186

Roenrig, G. H.; Kruse, R. A. y Kern, A. (2007). Teacher and School Characteristics and Their Influence on Curriculum Implementation. Journal of Research in Science Teaching, 44(7), pp. 883907.

http://dx.doi.org/10.1002/tea.20180

Schwarz, C. (2009). Developing Preservice Elementary Teachers' Knowledge and Practices through Modeling-Centered Scientific Inquiry. Science Education, 93(4), pp. 720-744.

http://dx.doi.org/10.1002/sce.20324

Shulman, L. S. (1985). Paradigms and research programs in the study of teaching: A contemporary perspective, pp. 3-36. En: M. C. Wittrock (ed.). Handbook of Research on Teaching. New Cork: MacMillan.

Singer, J.; Lotter, C.; Feller, R. y Gates, H. (2011). Exploring a Model of Situated Professional Development: Impact on Classroom Practice. Journal of Science Teacher Education, 22(3), pp. 203227. http://dx.doi.org/10.1007/s10972-011-9229-0

Tejada, J. (1999a). Acerca de las competencias profesionales I. Herramientas, 56, pp. 20-30.

TeJadA, J. (1999b). Acerca de las competencias profesionales II. Herramientas, 57, pp. 8-14.

Valcárcel, M. V. y Sánchez, G. (2000). La formación del profesorado en ejercicio, pp. 557-582. En: F. J. Perales y P. Cañal (eds.). Didáctica de las Ciencias Experimentales. Alcoy: Marfil. 


\title{
Reform of initial science teacher training: design proposal for a competency-based curriculum
}

\author{
F. Javier Perales Palacios, J. Manuel Cabo Hernández, J. Miguel Vílchez González, Manuel Fernández González, \\ Francisco González García, Pilar Jiménez Tejada \\ Dpto. de Didáctica de las Ciencias Experimentales. Universidad de Granada \\ fperales@ugr.es, jmcabo@ugr.es, http://www.ugr.es/ jmvilchez/Web/,mfgfaber@ugr.es, http://www.ugr.es/local/pagoga, \\ pjtejada@ugr.es
}

This article aims to establish a procedure for selecting the desirable competences for the current "University Master's Degree in Secondary School, Vocational Training and Language Teaching Teacher" recently introduced in Spain, and which is part of the new structure of Spanish Postgraduate studies and of the European Higher Education Space. Its purpose focuses specifically on the area of scientific knowledge (branches of Physics-Chemistry and Biology-Geology).

For this purpose, we have resorted to various sources that we considered relevant for the design of the curriculum, such as models of pre-service teacher training current in educational literature, the present curriculum of Science in Compulsory Secondary Education in Spain, the PISA International Program of Assessment, the opinion of in-service teachers, teachers' epistemological conceptions, teachers' professionalization and the demands it generates and, finally, the social dimension of science.

Based on previously established competences in the legal framework of the Master referred to, new or nuanced competences have been incorporated by way of criticism. Specifically, we begin with an analysis of the concept of competence and we extend it to that adopted by the Master referred to. Then we discuss the matter, based on the contributions from each of the sources we have used for the selection of competences:

- Models of pre-service teacher training. These usually choose training for a specific degree for future science teachers, which does not happen in our case.

- Current Science Curriculum in Compulsory Secondary Education. The legislation includes a specific competence called "Competence in knowledge and interaction with the physical world." Its descriptors were taken into consideration.

- PISA International Program. We considered the PISA (2006) report, since this edition was the last test with emphasis on sciences. To this aim, we looked for the competence indicators that the authors of this report assumed the students must possess and sought to assess and, where appropriate, we integrated them into the competences already available.

- In-service teachers' opinion. A questionnaire developed on line was presented as a Likert-type scale. The questionnaire contained questions about the competences acquired in initial training and then on the same skills in their current activity as teachers. We considered only those competences with a great difference of measurements between the use made of them in the classroom and the initial training received, as this implied a deficit in training that would require incorporation into the selection made.

- Teachers' epistemological conceptions. A review of the literature on teachers' epistemological conceptions was made. This was done given that these conceptions determine, to a large extent, not only the teachers' view of science, but what they convey in their teaching. This review enabled us to detect that a number of epistemological dimensions were not reflected in the previous list of competences, so they were added.

- Teaching professionalization. We returned to the concept of competence discussed at the beginning of the work to adapt it to the professional competence of future teachers and to visualize the shortcomings of the current training model. This led us to introduce specifications on the multicultural reality of schools, which also merit treatment in science classes and a competence related with classroom reality, essential for future career development. Furthermore, Sustainable Development has been incorporated as the integrating axis, with a holistic perspective and considering the interrelationships between the different dimensions of sustainability.

- Social Dimension of Science. This last section focuses on the role of science in the mass-media, as they are the nearest social reference to science students and future teachers, acting as mediators between 'global' scientific culture and the citizens' personal one. An analysis of the literature shows the general need to train future citizens for life, where the mass-media become privileged mediators between Science-Technology and Society. This involves introducing new competences or modification of the previous ones.

The paper ends with a proposal of the complete list of desirable competences for future science teachers in Secondary Education and an analysis of the innovative additions carried out in this process of curriculum design. 